\title{
Electronic real-time surveillance for influenza-like illness: experience from the 2009 influenza A(H1N1) pandemic in Denmark
}

K M Harder (katjaharder@gmail.com) ${ }^{1}$, P H Andersen ${ }^{1}$, I Bæhr ${ }^{1}$, L P Nielsen ${ }^{2}$, S Ethelberg ${ }^{1}$, S Glismann ${ }^{1}$, K Mølbak $^{1}$

1. Department of Epidemiology, Statens Serum Institut, Copenhagen, Denmark

2. Department of Virology, Statens Serum Institut, Copenhagen, Denmark

Citation style for this article:

Harder KM, Andersen PH, Bæhr I, Nielsen LP, Ethelberg S, Glismann S, Mølbak K. Electronic real-time surveillance for influenza-like illness: experience from

the 2009 influenza $A\left(\mathrm{H}_{1} \mathrm{~N}_{1}\right)$ pandemic in Denmark. Euro Surveill. 2011;16(3):pii=19767. Available online: http://www.eurosurveillance.org/ViewArticle.

aspx?Articleld $=19767$

Article published on 20 January 2011

To enhance surveillance for influenza-like illness (ILI) in Denmark, a year-round electronic reporting system was established in collaboration with the Danish medical on-call service (DMOS). In order to achieve realtime surveillance of ILI, a checkbox for ILI was inserted in the electronic health record and a system for daily transfer of data to the national surveillance centre was implemented. The weekly number of all consultations in DMOS was around 60,000, and activity of ILI peaked in week 46 of 2009 when $9.5 \%$ of 73,723 consultations were classified as ILI. The incidence of ILI reached a maximum on 16 November 2009 for individuals between five and 24 years of age, followed by peaks in children under five years, adults aged between 25 and 64 years and on 27 November in senior citizens (65 years old or older). In addition to the established influenza surveillance system, this novel system was useful because it was timelier than the sentinel surveillance system and allowed for a detailed situational analysis including subgroup analysis on a daily basis.

\section{Introduction}

In most industrialised countries, surveillance for influenza-like illness (ILI) is carried out by networks of sentinel general practitioners or clinics. Data from sentinel surveillance, in combination with virological data, constitute the basis for influenza surveillance, and has for many years proven to be of value [1]. However, the sentinel surveillance systems have limitations. In most countries, participation in the system is voluntary and it requires time and commitment for a general practitioner to report on a regular basis. Due to a limited number of active sentinel practitioners, analysis of trends and differences by subgroups such as age or geography may also be imprecise. Furthermore, reporting from sentinel practitioners is often done on a weekly basis and only during the influenza season. Finally, the Danish sentinel system, as organised at the present, has delays due to mail delivery from the sentinel practices to the surveillance institute and other practicalities $[2,3]$.

To enhance influenza surveillance, a year-round simple electronic reporting system was established in
Denmark in collaboration with the Danish medical oncall service (DMOS). Nearly real-time surveillance of ILI was achieved by a simple checkbox for ILI inserted in the electronic health record. This system was first established in 2006 and covered the entire country in 2008. This paper describes the DMOS surveillance system and reports data from the influenza $\mathrm{A}\left(\mathrm{H}_{1} \mathrm{~N}_{1}\right)_{2} 009$ pandemic from May 2009 to January 2010 where this surveillance system allowed a risk assessment of ILI trends on a daily basis.

\section{Methods}

DMOS is a national public medical service replacing the function of the general practitioners after opening hours. On weekdays, this service is open for attendance from $4 \mathrm{pm}$ to $8 \mathrm{am}$, and during weekends and national holidays on a 24 -hours basis. The service is staffed by physicians, mainly general practitioners. DMOS can only be contacted by telephone. The duty officer will either give advice on the phone, make an appointment for a consultation (at the nearest public clinic staffed by DMOS or a home visit, depending on the circumstances), or refer for admission to hospital.

All contacts are registered in a single national computer system. In the electronic health record, demographic data are registered in a structured format, but the medical history, diagnosis and actions taken are recorded in a free text format. In agreement with the on-call physicians and the Danish Medical Association, the computer system was in 2006 modified when a checkbox for ILI was added in the userinterface of the data system. It has a 'mouse-over' function presenting the ILI definition. When the ILI checkbox is marked, the following text with the ILI definition is automatically entered in the unstructured text field: 'Influenza-like illness (ILI): sudden onset of fever, muscle pain, headache and respiratory symptoms'. The cursor is placed after this text, and the physician may enter additional clinical information. With this simple improvement it became possible to obtain structured data on ILI without interfering with the routines of the physicians. In our definition of ILI all three symptoms must be present in order to increase the specificity of the diagnosis. 
On a real-time basis, data are transferred to a common external server. On working days, a surveillance data extract is transferred daily to the national public health institute for infectious diseases (Statens Serum Institut). Data are available before $1 \mathrm{pm}$. The file uploaded on Monday includes activities from Friday, 4 pm to Monday, 8 am.

The data file contains the following information on each contact: time of contact, ILI (yes/no), age in

\section{FIGURE 1}

Contacts to the on-call medical service and influenza-like illness cases, per week, Denmark, 2008-2010

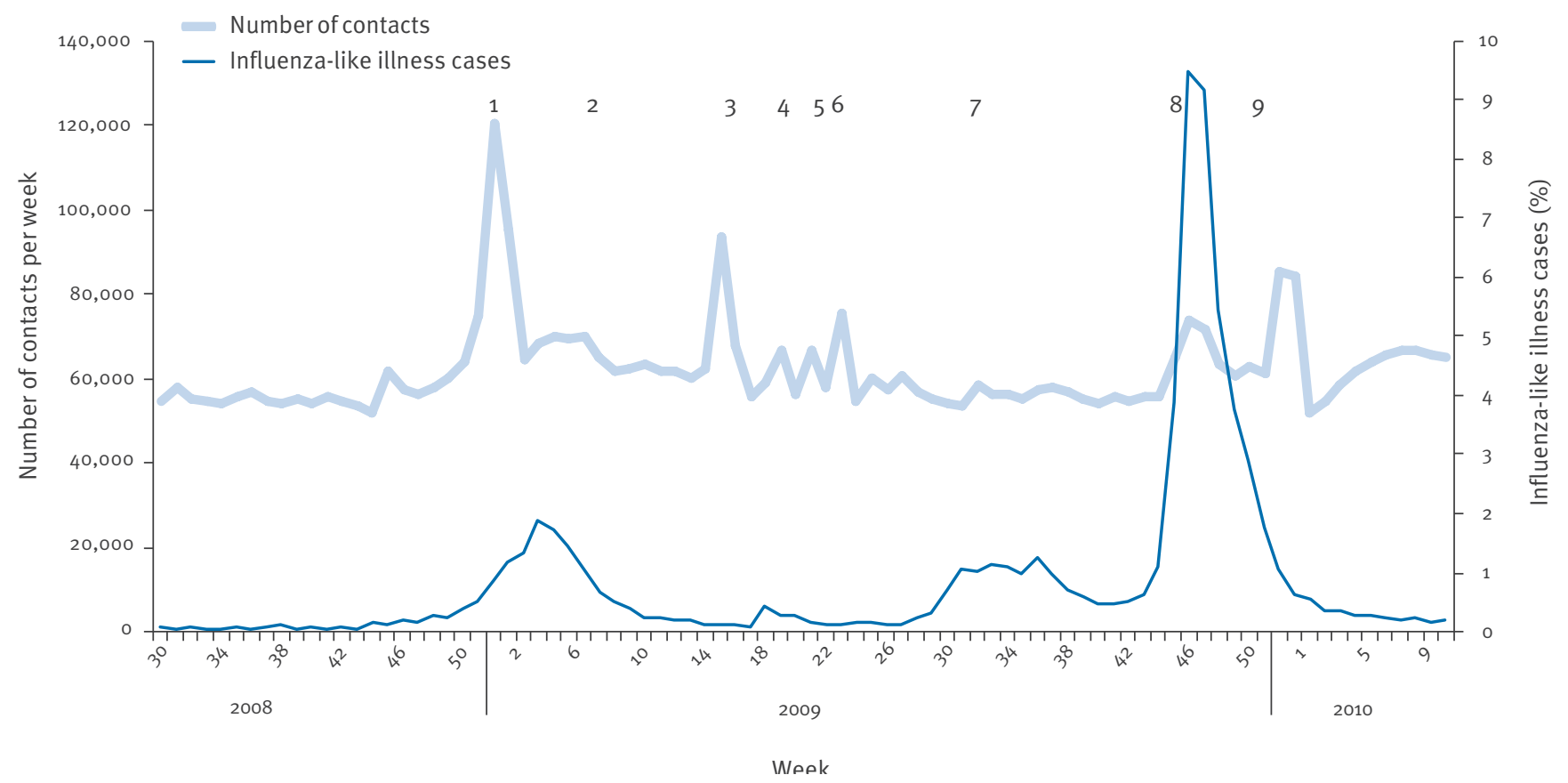

1: Christmas 2008; 2: Seasonal influenza 2008/09; 3: Easter 2009; 4-6: Other public holidays; 7: Summer wave of the influenza A(H1N1)2009 pandemic; 8: Autumn wave of the influenza A(H1N1)2009 pandemic; 9: Christmas 2009.

\section{FIGURE 2}

Age-specific incidence of influenza-like illness cases per day, medical on-call service, Denmark, 15 October 20 December 2009

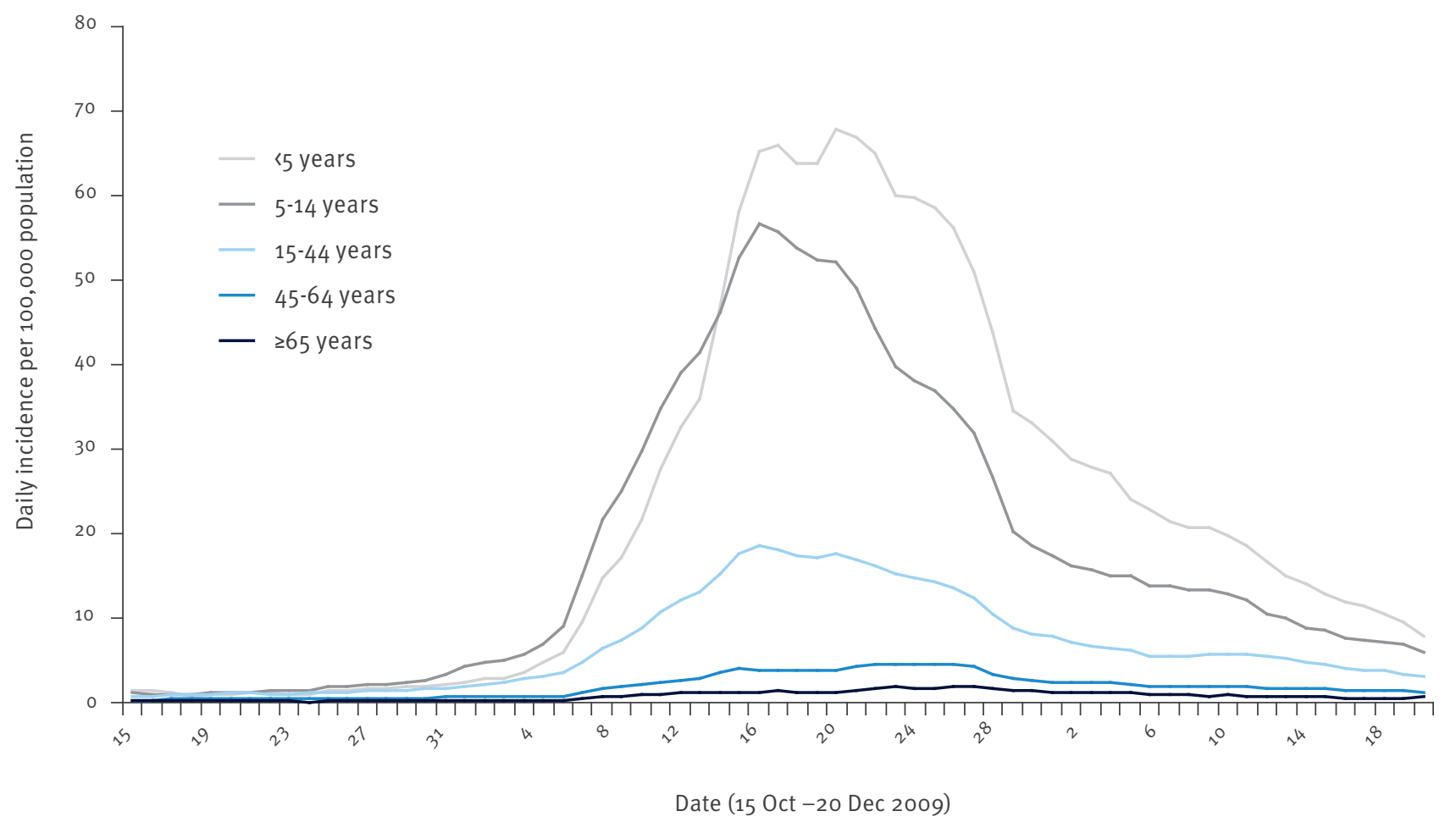


months, sex, residence of patient (postal code), geographical region of the reporting DMOS physician, type of contact: call, followed by consultation, doctor's visit to the home of the patient, or hospital admission. When a patient contacts the on-call service more than once during one working period, only one record is generated and the information on action taken is the last action taken (e.g. visit to a clinic or admission to hospital). No personal information on individuals is transferred through this system.

At Statens Serum Institut, data are stored in a SQL database and analysed to obtain the incidence rate of ILI and the proportion of patients with ILI of all patients managed (consultation percentage). The results are analysed by age group and geographical region. During the peak influenza period, a seven-day moving

\section{TABLE}

Referral of patients with influenza-like illness to consultation at a clinic or hospital during seasonal influenza 2008/09 and summer and autumn waves of influenza A(H1N1)2009, Denmark, 2008-2010

\begin{tabular}{|c|c|c|c|}
\hline \multirow{2}{*}{ Period } & \multicolumn{2}{|c|}{ Patients with influenza-like illness } & \multirow{2}{*}{ Relative risk $(95 \% \mathrm{Cl})^{d}$} \\
\hline & Total & Referred to consultation, Number (\%) & \\
\hline Seasonal influenza ${ }^{a}$ & 9,158 & $4,321(47)$ & $1 \quad$ (reference) \\
\hline Autumn wave & 29,735 & $8,390(28)$ & $0.62(0.60$ to 0.64$)$ \\
\hline
\end{tabular}

$\mathrm{Cl}$ : confidence intervals.

a 8 December 2008 to 15 March 2009.

b 13 July to 11 October 2009.

' 12 October 2009 to 18 April 2010.

${ }^{\mathrm{d}}$ Adjusted for age by Poisson regression analysis.

Source: Danish medical on-call service.

\section{FIGURE 3}

Weekly incidence of influenza-like illness cases, Denmark, 2009-2010

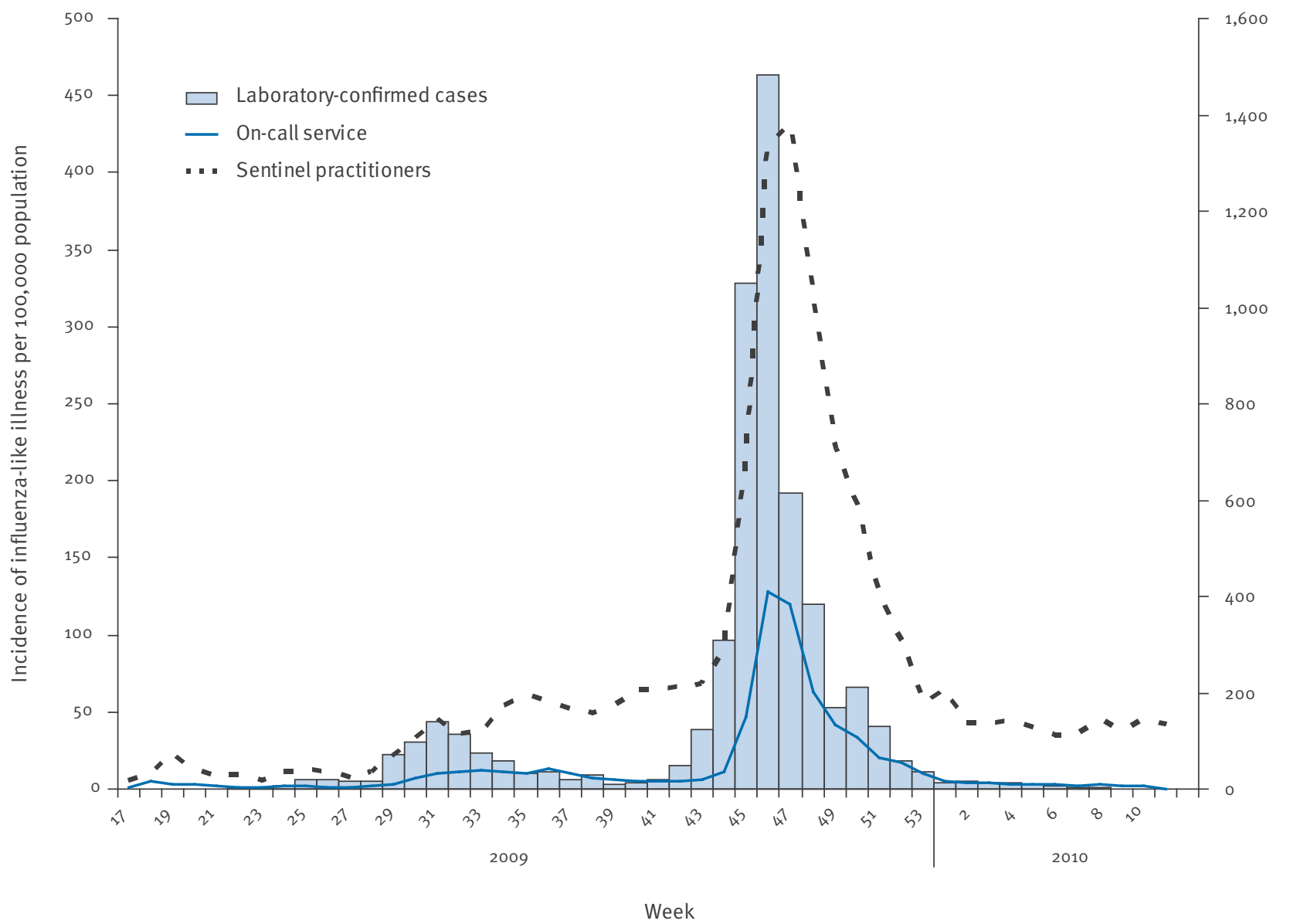

The left $y$-axis represents cases recorded by the Danish medical on-call service.

The right $y$-axis represents the number of laboratory-confirmed infections with influenza $A\left(\mathrm{H}_{1} \mathrm{~N}_{1}\right) 2009$ virus. 
average was presented daily on the website of Statens Serum Institut. Furthermore, a weekly report based on data aggregated over a full week were presented along with data from sentinel surveillance and virological data from the weekly influenza bulletin published every Wednesday on the Statens Serum Institut website. Because the system was recently implemented, we have not yet established a historical baseline and epidemic thresholds for these outcome measures.

The data were compared by visual inspection with national data of laboratory-confirmed influenza $A\left(\mathrm{H}_{1} \mathrm{~N}_{1}\right)_{2009}$ and with data from the sentinel surveillance which during the autumn comprised information from approximately 250 general practitioners. We calculated the number of calls that were followed by referral to a consultation (defined as consultation at a public clinic, doctor's visits to patients' homes, or hospital admission), and compared the proportion of calls that resulted in a consultation between ILI registered during the periods of influenza $A\left(\mathrm{H}_{1} \mathrm{~N}_{1}\right)_{2009}$ transmission and seasonal influenza in the season 2008/09 ('referral rates'). Because patients were younger in the influenza $A\left(\mathrm{H}_{1} \mathrm{~N}_{1}\right) 2009$ pandemic than in seasonal influenza, the referral rates were adjusted for age by Poisson regression (age in five-year groups as categorical variables). We used the GENMOD procedure of the SAS statistical software (SAS institute, Cary, NC, United States of America).

We developed an application available on the website of Statens Serum Institut showing the spatial distribution of ILI in Denmark and the timeline of the pandemic [4]. A geographic information system (GIS) was applied to show the temporal-spatial development of ILI cases as well as the proportion of consultations with ILI diagnosis. Graduated colours of regions were used to show the proportion of consultations based on DMOS location and proportional circles were used to indicate the number of cases per geographic unit (post districts) based on the home address of the patients. The ILI activity monitored by the DMOS was reported to the public on the website of Statens Serum Institut and the Danish public service broadcasting company (Danmarks Radio) on a weekly basis with ILI incidence graphics and maps of ILI incidence in different regions of Denmark. Geographic maps were produced with ArcGIS 9.3, ESRI and the time graphic with Emprise JavaScript ChartsTM, Emprise Corporation.

In this paper, we report data from calendar week 30 of 2008 (starting on 21 July 2008) to week 15 of 2010 (last day included is 18 April 2010). The dataset contained information on about 5.7 million contacts over 91 weeks.

\section{Results}

The median weekly number of contacts to the DMOS was 60,029 corresponding to 1,089 contacts per 100,000 population. Peak activities were seen around winter holidays (with a maximum of 120,535 contacts in week 52 of 2008 and 95,080 in week 1 of 2009), Easter $(96,586$ contacts in week 13 of 2009) and in the Danish public holidays that follow Easter (Figure 1).

The proportion of cases with ILI ranged from $0.05 \%$ in week 30 of 2008 to $9.5 \%$ in week 46 of 2009 , which coincided with the peak of the autumn wave of the influenza $A\left(\mathrm{H}_{1} \mathrm{~N}_{1}\right) 2009$ pandemic. In the peak week, 6,987 of 73,723 contacts were classified as ILI. Increase in the proportion of ILI cases was additionally seen during periods with seasonal influenza in the beginning of 2009 (maximum $1.9 \%$ in week 3, 2009). A peak in ILI activity was also noted in the late summer of 2009 when cases of influenza $A\left(\mathrm{H}_{1} \mathrm{~N}_{1}\right) 2009$ were imported to Denmark, but only limited domestic transmission occurred. In this summer wave, a maximum activity of $1.3 \%$ was observed in week 36 of 2009.

Figure 2 shows the daily age specific incidence (sevenday moving average) of ILI in the period from 15 October to 20 December 2009. Age specific peaks appeared from 16 to 27 November 2009 (weeks 47 and 48).

In children aged between five and 14 years, the incidence increased from 0.9 per 100,000 population $(n=6)$ on 17 October to a peak of 57 per 100,000 population $(n=387)$ on 16 November 2009. On the same day, there was a peak in the incidence of cases among individuals aged between 15 and 24 years (18 per 100,000 population, $n=396)$. The incidence in children under five years of age peaked on 20 November (68 per 100,000 population, $n=222$ ), in adults aged between 25 and 64 years on 24 November (5 per 100,000 population, $n=68$ ), and persons aged 65 years or more on 27 November ( 2 per 100,000 population, $n=17$ ).

In order to examine referral rates, the data were analysed according to three time periods determined according to influenza transmission: seasonal influenza (8 December 2008 to 15 March 2009), influenza A (H1N1)2009 summer wave (13 July to 11 October 2009), and autumn wave (from 12 October 2009 to 18 April 2010) (Table).

Referral rates were highest for seasonal influenza ( $47 \%$ ), whereas only $26 \%$ and $28 \%$ were referred for consultation during the two pandemic waves. Patients were younger in the autumn wave of the pandemic than in the seasonal influenza period: median age (interquartile range) was 27 years (11 to 41 years) in the seasonal influenza period, 27 years (15 to 40 years) in the summer peak and 15 ( 6 to 32 years) in the autumn peak. We therefore adjusted for age by Poisson regression and time period remained independently associated with referral rate (Table).

Figure 3 shows overall incidence of ILI in the sentinel practices (adjusted for number of reporting sentinel practices), incidence of ILI in DMOS as well as the number of laboratory-confirmed cases of influenza 
$A\left(\mathrm{H}_{1} \mathrm{~N}_{1}\right) 2009$ reported to the Department of Virology, Statens Serum Institut.

The incidence of ILI was higher in the sentinel system than in the DMOS. In both systems, marked increases in incidence were observed in week 45 and the peak appeared a week earlier in the DMOS compared with the sentinel surveillance. Thus, the peak incidence in DMOS was in week 46 of 2009 with 128 cases per 100,000 population whereas the peak incidence in the sentinel system was 432 cases per 100,000 population in week 47. The latter estimate was based on 1,864 reports from 288 practices extrapolated to the total of 3,655 general practitioners in Denmark. For comparison, the incidence of laboratory-confirmed cases of influenza $A\left(\mathrm{H}_{1} \mathrm{~N}_{1}\right) 2009$ peaked in week 46 with 1,472 cases ( 27 cases per 100,000 population).

\section{Discussion}

During the 2009 pandemic, the DMOS provided valuable real-time and detailed information on ILI-incidence in different age groups and geographical areas. The surveillance data were updated each week. However daily updates were used during the autumn wave of the pandemic, as illustrated in Figure 2. This enabled us to provide timely data to policy makers and health authorities. In particular, they were able to get an overview of the influenza activity during the previous day whereas the sentinel system had more than a week delay. To our knowledge, this is the first year-round, real-time electronic syndromic influenza surveillance system with national coverage that is based on reports provided by physicians. The surveillance system had several advantages among which the automatic data transfer and the daily reporting were the most important. The fact that it was added to an existing administrative system, made it simple to establish and maintain and can therefore be considered as an efficient approach to syndromic surveillance.

Other systems for influenza surveillance, including traditional surveillance for consultation of general practitioners for ILI or acute respiratory infections within their working hours, ambulance dispatches $[5,6]$ and hospital admissions [7,8], may in emergencies or in times of lack of resources become 'saturated'. It is obvious that such systems have limited capacity (for instance, the number of ambulance dispatches will be limited by the number of ambulances and ambulance drivers, and people will find alternative ways to get to hospital during crisis). General practitioners often have a very busy schedule of planned visits and may only have a small number of slots open for acute illnesses. By contrast, the public on-call service is more flexible. There are by definition no planned visits and capacity may be increased by calling in standby medical doctors and adding more telephone lines. This may be one of the reasons that the signal from the on-call service came earlier than in the sentinel surveillance (Figure 1). However, it is also possible that there are differences in the characteristics of the patients (including age) who use the two systems and that this contributes to a later peak in the sentinel system. Importantly, we were able to demonstrate that the peak in the virological surveillance corresponded well with the peak in the DMOS system.

Another possible useful source for influenza surveillance are web queries $[9,10]$. Web queries have the advantage of being cost-effective and timely and may serve as an early indication of unusual activity. However, since they are based on lay reporting, data are more subjective than the present system which has both the advantage of being very timely and automated while still based on evaluation by medical staff. An interesting development of influenza surveillance is Gripenet and related surveillance schemes consisting of cohorts of volunteers reporting ILI cases on a regular basis on the Internet [11]. Gripenet is a fast and flexible monitoring system whose uniformity allows for direct comparison of ILI rates between countries and is useful for assessing the burden of illness. However, it requires more commitment from administrative staff and participants than does DMOS system and cases are not evaluated by medical staff.

Nevertheless, the DMOS system has its limitations. As opposed to the sentinel system, there are no virological data from the on-call physicians. Therefore, it cannot replace the sentinel system. Furthermore, sentinel doctors are committed to influenza surveillance, whereas the on-call service is staffed by a larger group of physicians with different knowledge and attitude towards influenza surveillance. Although the novel system was promoted in the regions that administer the DMOS, we have no formal evaluation of its use and the completeness of reporting.

The emergence of influenza $A\left(\mathrm{H}_{1} \mathrm{~N}_{1}\right)_{2009}$ outside the normal 2009/10 influenza season, the high morbidity, the high burden of illness in children and young adults, and the occurrence of several waves are all characteristics of a pandemic [12]. The system described here was sufficiently sensitive to be able to detect different peaks for different age groups, and we hope that such detailed data will be of value to obtain more detailed knowledge on the pandemic. As shown in the Table, patients with pandemic influenza were less frequently referred to consultation or admitted to hospital than patients with seasonal influenza in the 2008/09 season. This confirms that in most patients, the clinical presentation in the 2009 pandemic was mild [13-15], but may also reflect that the public may have been concerned with the situation and that the threshold for contacting the healthcare system was lower than in periods with seasonal influenza, with the on-call physicians being the most accessible professionals. From July 2009, the Danish National Board of Health advised the public to use the telephone for getting in contact with the healthcare system and to restrict physical consultations in order to limit the spread of influenza 
$A\left(\mathrm{H}_{1} \mathrm{~N}_{1}\right)$ 2009. A relatively low referral rate may reflect that this advice was often followed [16].

In conclusion, we established a simple, yet comprehensive and timely, system that allowed us to follow the incidence and consultation percentage of ILI during the autumn of 2009 when pandemic influenza peaked in Denmark. The system allowed for a detailed situational analysis and was useful for the health authorities' response to the pandemic, including risk communication. We propose that other countries explore the possibility of establishing such a system which may also be of relevance for other public health threats.

\section{Acknowledgements}

We acknowledge the excellent collaboration with the Danish Medical Association and the Danish on-call physicians, and the contributions of Annette Hartvig Christiansen, Karina Lee Petersen, Linda Roth and Marianne Hauge Jensen.

\section{References}

1. Paget J, Marquet R, Meijer A, van der Velden K. Influenza activity in Europe during eight seasons (1999-2007): an evaluation of the indicators used to measure activity and an assessment of the timing, length and course of peak activity (spread) across Europe. BMC Infect Dis. 2007;7:141.

2. Dailey L, Watkins RE, Plant AJ. Timeliness of Data Sources Used for Influenza Surveillance. I Am Med Inform Assoc. 2007;14(5):626-31.

3. Coory M, Grant K, Kelly H. Influenza-like illness surveillance using a deputising medical service corresponds to surveillance from sentinel general practices. Euro Surveill. 2009;14(44): pii=19387. Available from: http://www. eurosurveillance.org/ViewArticle.aspx?Articleld=19387

4. Statens Serum Institut. ILI Surveillance based on DMOS reporting system. Copenhagen:Statens Serum Institut. [Accessed 20 Jan 2011]. Available from: http://www.ssi.dk/ graphics/DMOS/index.html

5. Bork KH, Klein BM, Mølbak K, Trautner S, Pedersen UB, Heegaard E. Surveillance of ambulance dispatch data as a tool for early warning. Euro Surveill. 2006;11(12):pii=669. Available from: http://www.eurosurveillance.org/ViewArticle. aspx?Articleld $=669$

6. Coory MD, Kelly H, Tippett V. Assessment of ambulance dispatch data for surveillance of influenza-like illness in Melbourne, Australia. Public Health. 2009;123(2):163-8.

7. Hadler JL, Siniscalchi A, Dembek Z. Hospital admissions syndromic surveillance--Connecticut, October 2001-June 2004. MMWR Morb Mortal Wkly Rep. 2005;54 Suppl:169-73.

8. Zurynski YA, Lester-Smith D, Festa MS, Kesson AM, Booy R, Elliott EJ. Enhanced surveillance for serious complications of influenza in children: role of the Australian Paediatric Surveillance Unit. Commun Dis Intell. 2008;32(1):71-6.

9. Johnson HA, Wagner MM, Hogan WR, Chapman W, Olszewski RT, Dowling J, et al. Analysis of web access logs for surveillance of influenza. Stud Health Technol Inform. 2004;107(Pt 2):1202-6.

10. Hulth A, Rydevik G, Linde A. Web queries as a source for syndromic surveillance. PLoS One. 2009;4(2):e4378. Epub 6 Feb 2009.

11. van Noort SP, Muehlen M, Rebelo de Andrade H, Koppeschaar C, Lima Lourenço JM, Gomes MG. Gripenet: an internetbased system to monitor influenza-like illness uniformly across Europe. Euro Surveill. 2007;12(7):pii=722. Available from: http://www.eurosurveillance.org/ViewArticle. aspx?Articleld $=722$

12. Miller MA, Viboud C, Balinska M, Simonsen L. The signature features of influenza pandemics--implications for policy. $\mathrm{N}$ Engl J Med. 2009;360(25):2595-8.

13. Donaldson LJ, Rutter PD, Ellis BM, Greaves FE, Mytton OT, Pebody RG, et al. Mortality from pandemic A/H1N1 2009 influenza in England: public health surveillance study. BMJ. 2009;339: b5213.
14. Nicoll A, McKee M. Moderate pandemic, not many dead-learning the right lessons in Europe from the 2009 pandemic. Eur J Public Health. 2010;20(5):486-8.

15. Wielders CC, van Lier EA, van 't Klooster TM, van GageldonkLafeber AB, van den Wijngaard CC, Haagsma JA, et al. The burden of 2009 pandemic influenza $A\left(\mathrm{H}_{1} \mathrm{~N}_{1}\right)$ in the Netherlands. Eur J Public Health. 2010. [Epub ahead of print]

16. Sundhedsstyrelsen. Influenza $A\left(\mathrm{H}_{1} \mathrm{~N}_{1}\right) v$ - Clarification of new guidelines. EPI-NEWS. 2009;27-29. Available from: http:// www.ssi.dk/English/News/EPI-NEWS/ /media/Indhold/ EN\%20-\%20engelsk/EPI-NEWS/2009/pdf/EPI-NEWS\%20-\%20 $2009 \% 20-\% 20 \mathrm{No} \% 2027-29$. ashx 